\title{
Efficacy of Thunder-Fire Moxibustion In Treating Ankylosing Spondylitis of Kidney Deficiency And Governor Meridian Cold And Its Influence On Bone Metabolism凶Study Protocol For A Randomized Controlled Trial
}

\section{Yang Liu}

Beijing Luhe Hospital Affiliated to Capital Medical University

Pei Wang

Beijing Luhe Hospital Affiliated to Capital Medical University

\section{Yanyan Sun}

Beijing Luhe Hospital Affiliated to Capital Medical University

Jing Qu

Beijing Luhe Hospital Affiliated to Capital Medical University

Min Li ( $\sim 13810048883 @ 139 . c o m)$

Beijing Luhe Hospital Affiliated to Capital Medical University https://orcid.org/0000-0002-9171-4169

\section{Research Article}

Keywords: Ankylosing spondylitis(AS), Thunder-Fire Moxibustion,Kidney Deficiency and Governor Meridian Cold, Bone Metabolism, randomized controlled trial

Posted Date: September 22nd, 2021

DOI: https://doi.org/10.21203/rs.3.rs-559722/v1

License: (c) (i) This work is licensed under a Creative Commons Attribution 4.0 International License. Read Full License 


\section{Abstract}

Background: Ankylosing spondylitis(AS) is a common chronic inflammatory spondyloarthropathy,.It is considered in Traditional Chinese Medicine(TCM) that the pathogenesis of AS is mainly due to Yang deficiency of kidney governor meridian and internal prosperity of cold evil. Thunder-fire moxibustion is a kind of moxibustions and characterized in abundance in drug composition, high heat radiation and strong penetration. Thunder-fire moxibustion on spinal segment of governor meridian in treating AS seems compatible with the main pathogenesis of kidney deficiency and governor meridian cold. The Trial aims to explore the efficacy of thunder-fire moxibustion in patients with AS of kidney deficiency and governor meridian cold and its influence on bone metabolism, through a prospective randomized trial.

Methods: Sixty patients with AS of kidney deficiency and governor meridian cold will be recruited and randomly assigned to the treatment group \thunder-fire moxibustion three times a week plus basic treatment\and the control group (basic treatment) at the Center of TCM of Beijing Luhe Hospital Affiliated to Capital Medical University (Beijing, China), Each patient will be treated for 4 weeks.Primary outcome indexes will include efficacy of TCM syndrome,Bath Ankylosing Spondylitis Disease Activity Index (BASDAl), Bath Ankylosing Spondylitis Functional Index (BASFI), with observation once respectively prior to the treatment, 2 weeks post to the treatment, and at the end of the treatment. Secondary outcome indexes will include tumor necrosis factor- $a(T N F-a)$ and receptor activator of nuclear factor-k $B$ ligand(RANKL), which will be determined once respectively before and after treatment.Side effects during the treatments will be recorded and analyzed as well. Inter-group comparison and analysis will be performed based on intention-to-treat set and per-protocol set.

Discussion: This prospective randomized trial will help verify the efficacy of thunder-fire moxibustion in treating AS of kidney deficiency and governor meridian cold, discuss preliminarily its mechanism in treating this disease, and provide high-quality evidences for scientific researches on clinical treatment with thunder-fire moxibustion against AS.

Trial registration: Chinese Clinical Trial Registry, ChiCTR2100044227 . Registered on 12 March 2021.

\section{Introduction}

\section{Background and rationale\{6a\}}

Ankylosing spondylitis (AS), a common chronic and inflammatory spondyloarthropaty, is characterized by sacroiliac joint pain and lumbodorsal morning stiffness with limited movement in the early stage. Patients with the severe disease may manifest hip joint damage, spinal ankylosis and deformity, which are the main factors predisposing the sufferers to disabilities[1,2]. It has been found by epidemiological researches that the incidence of AS in ordinary populations is about $0.09-0.3 \%$, with a certain difference in various countries related possibly to source of data or discrepancy in diagnostic criteria for this disease[3]. The incidence of AS in China is about 0.3\%[4]. Due to the complexity of AS with a high misdiagnosis rate in the early stage and a high rate of disability in the advanced stage, it may induce 
lesions in various degrees in multiple systems or even incapacitate a sufferer, influence the quality of life in a case of a severe disease with a great psychological and economic burden on the patient, and has become gradually a serious social problem.

There is no radical cure available for AS now, and the treatment aims mainly to reduce pain, delay the progress of the disease, and improve the quality of life. In the process of treatment, patients often prefer TCM, especially the external treatment of TCM with minor side effects and great human convenience. It is of great importance, therefore, for a medical practitioner to find an effective, safe and green therapy.

Moxibustion is a traditional treatment in TCM, using moxa or other agents for cauterizing or warmly ironing specific sites on human body, as stated in The Yellow Emperor's Canon of Internal that fire should be indicated for treatment of either Yin and Yang deficiency. It is considered in TCM that moxibustion is based on meridian doctrine, and Qi got by meridians post to moxibustion may warm the body surface and is transmitted downward through the acupoints along meridians, thereby achieving the effects in warming meridians, dispersing cold, promoting Qi, activating blood circulation, and balancing Yin and Yang[5]. Its mechanism might be related to thermal effect, radiation effect and pharmacological function of moxibustion[6]. It is illustrated by modern medicine[7,8,9]that moxibustion has the definite efficacy in regulating immunity, resisting inflammation/killing the pain, improving bone metabolism and maintaining bone mineral density.

A great deal of researches[10,11] either domestic or foreign in recent years has proven that moxibustion is an important approach to treat AS, with the techniques against this disease including spreading, partition, warmth and heat sensitivity, all of which have thermal stimulus despite their difference in manipulating methods and components. Thunder-fire moxibustion, one of moxibustions, has the advantages such as richer components of drugs, higher temperature, greater thermal radiation, stronger penetration[12], in comparison with ordinary moxibustions. Certain efficacy has been identified by researches[13] for thunder-fire moxibustion in treating pain and osteoarthropathy. Its indication in treating AS, therefore, is supported by evidences, despite fewer high-quality studies have been reported on the application of thunder-fire moxibustion to the treatment of AS.

\section{Objectives $\{7\}$}

In this research, we aim to verify the clinical efficacy of thunder-fire moxibustion in treating AS, through analyzing responses and influences on inflammation and bone metabolism in AS patients treated by thunder-fire moxibustion plus medication in comparison with those treated by medication alone, thereby discussing preliminarily the effects of thunder-fire moxibustion on arthritis and abnormal bone metabolism, and providing a theoretical basis for studies on thunder-fire moxibustion applied to AS treatment and the clinical generalization for this indication.

\section{Trial design $\{8\}$}


The Study is designed as a prospective, single-center, randomized and controlled clinical trial.Procedures in the trial lasting six weeks in total will include a 2-week adaption for subjects prior to randomization, followed by a 4-week treatment using a basic medication either alone or in combination with thunder-fire moxibustion.

\section{Methods:participants, Interventions, And Outcomes}

\section{Study setting $\{9\}$}

The trial will be carried out in Center of TCM in Beijing Luhe Hospital Affiliated to Capital Medical University (Beijing China). Patients are recruited in the TCM clinic and rheumatism immune clinic of Beijing Luhe hospital Affiliated to Capital Medical University.Patients are considered for inclusion if they meet the criteria as defined below.

\section{Eligibility criteria $\{10\}$}

\section{Primary inclusion criteria}

Patients must meet the following criteria to be eligible for the study:

$\triangle$ Meeting diagnostic criteria of both modern medicine and TCM;

$\nabla$ No exposure to any treatment within one month, or treated only with routine drugs and without any other external treatment available;

$\nabla$ Aged between 18 and 70 years; and

$\triangle$ Signing ICF based on voluntary acceptance of treatment, with good compliance to observation and examination.

\section{Primary exclusion criteria}

If the patients meet any of the following criteria at the screening visit, they will not be eligible for the study:

$\triangle$ Aged below 18 years or above 70 years; pregnant or lactating women; or intrinsic allergy or allergic to the drug in the Study;

\Complications such as severe primary diseases in cerebrovascular system, cardiovascular system, liver, kidneys and hemopoietic system, or a patient with a psychopath.

$\nabla A$ sufferer of arthritis in advanced stage, with severe deformity, rigidity and loss of labor force;

$\triangle$ Anybody meeting the inclusion criteria but failing in completing the treatment as specified, making the response unevaluable; or anybody with insufficient data affecting the assessment of efficacy or safety; 


\section{Who will take informed consent? $\{26 a\}$}

Patients with AS will be screened for eligibility to participate in this study based on the abovementioned criteria. Then they will receive initial study information. After at least 2 weeks of reflection, patients are invited to meet with the research physician to discuss any remaining questions and sign the informed consent.

\section{Interventions}

\section{Intervention description $\{11 \mathrm{a}\}$}

Thunder-fire moxibustion will be performed three times a week successively for 4 weeks with 12 times in total.The sites for thunder-fire moxibustion will be selected along the governor meridian in its spinal segment, with the acupoints Dazhui and Yaoshu as the border. All acupoints will be located according to National Standard of the People's Republic of China: Name and Location of Acupoints (GB/T 123462006 ) issued in 2006, where in acupoint Dazhui (GV14) is located in the infraspinous depression of the 7th cervical vertebra along the posterior middle line within the spinal region; acupoint Yaoshu (GV2) is at a place along the posterior middle line within the sacral region and opposite to sacral hiatus. See Figure 1.

The equipment for thunder-fire moxibustion includes thunder-fire moxa stick $(28 \mathrm{~mm} \times 105 \mathrm{~mm}$, manufactured by Chongqing Zhao's Thunder-Fire Moxibustion Traditional Medicine Research Institute), and the moxibustion box with 9 rear holes (provided by moxibustion room of TCM center of Beijing Luhe Hospital Affiliated to Capital Medical University ).

The operational methods of thunder-fire moxibustion is as follows: the patient is kept in prone position, and the selected acupoints are exposed. The moxibustion medicine fixed in small holes of the moxibustion box is ignited, and the box is placed onto the position of the governor meridian from acupoint Dazhui to that of Yaoshu at the back, where is covered with a towel for a warm moxibustion for 30 minutes. The medicine is removed once every 15 minutes to blow away the ashes. The moxibustion is continued until redness appears on the skin and deep tissue becomes warm, with local perspiration.

\section{Explanation for the choice of comparators $\{6 \mathrm{~b}\}$}

The control group receives basic treatment, sulfasalazine combined with celecoxib will be used. Sulfasalazine enteric coated tablets (manufactured by Shanghai Zhongxisunve Pharmaceutical Co., Ltd; national medicine permission number (NMPN)) 1.0g b.i.d. po; in combination with celecoxib capsule (repacked by Pfizer Pharmaceuticals; NMPN: J20140072) 200mg q.d. po. The treatment group receives thunder-fire moxibustion three times a week plus basic treatment.

\section{Criteria for discontinuing or modifying allocated interventions $\{11 \mathrm{~b}\}$}


Participation is voluntary;Participants will be withdrawn from the study at any time for any reason.Criteria for orderly discontinuing a participant's involvement in the study include the following:(A) the participant voluntarily withdraws from the study,(B) the participant does not complete all procedures/visits per protocol, (C) there are changes in their health status that makes continued participation inadvisable, (D)serious adverse events due to treatment.There will be no pre-defined criteria for modifying the allocated interventions.

\section{Strategies to improve adherence to interventions $\{11 \mathrm{c}\}$}

We plan to hold events to spread awareness about the treatments, which will encourage patient in volvement. .we will also keep in close contact with the participants if necessary throughout the study period.

\section{Relevant concomitant care permitted or prohibited during the trial $\{11 \mathrm{~d}\}$}

During the study protocol, participants will be asked to not receive any other external therapy in TCM,and all external therapy in TCM is not permitted 1 month before the conduct of the trial.

\section{Provisions for post-trial care $\{30\}$}

In the event of study-related damage or injuries, Beijing Luhe Hospital Affiliated to Capital Medical University shall provide compensation, except for claims that arise from misconduct or gross negligence of involved study personnel.

\section{Outcomes $\{12\}$}

\section{Primary outcomes}

The primary outcomes will include efficacy of TCM syndrome,Bath Ankylosing Spondylitis Disease Activity Index (BASDAI), Bath Ankylosing Spondylitis Functional Index (BASFI), with observation once respectively prior to the treatment, 2 weeks post to the treatment, and at the end of the treatment.

The efficacy of TCM syndrome will be evaluated by the TCM syndrome score(TCMSS) and TCM syndrome effective rate as the standard assessment. With the TCMSS, TCM syndrome is determined by stiffness and pain of the lumbar,sacro,ack,cervical spine and hip,fatigue and chills (See more details in Additional file 1).The scores range from 0 to 14. Then we will calculate the relative TCMSS by using the nimodipine method $\triangle T C M S S b$ stands for the TCMSS before treatment and TCMSSa is the TCMSS after

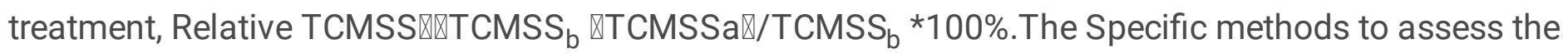

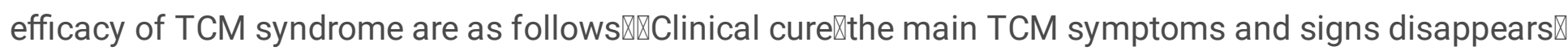

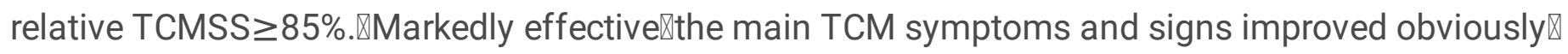

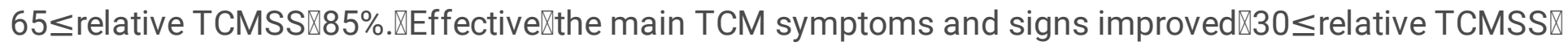

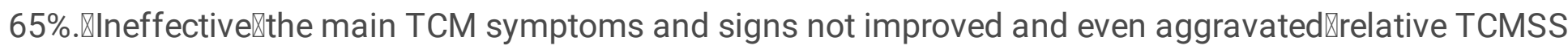
$₫ 30 \%$.Counting the number of patients who were clinically cured and markedly effective,and denoted by 
$\mathrm{n}_{1}$ and $\mathrm{n}_{2}$.Calculating The TCM syndrome effective rate using the following formula:TCM syndrome

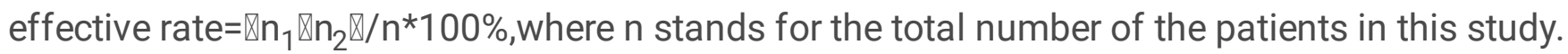

BASDAI and BASFI can reflect the patient's condition more comprehensive,which will be evaluated according to guideline formulated by International Ankylosing Spondylitis Evaluation Working Group in 2001 (See more details in See more details in Additional file 2 and 3). The BASDAl will be used to assess the disease activity and includes items on fatigue degree, pain or swelling degree of neck.back,hip and other joints, tendinitis, morning stiffness degree and duration of morning stiffness. The scores range from 0 to 10.The BASFI will be used to determine the functional status of patients' daily activities, which includes dressing,picking things up or taking things down,standing up from an armless seat or the floor, standing,climbing and doing physical activities. The scores range from 0 to 10 .

\section{Secondary outcomes}

Secondary outcomes including tumor necrosis factor- $\mathrm{a}$ (TNF- $\mathrm{a}$ ) and receptor activator of nuclear factor- $\mathrm{K}$ $B$ ligand(RANKL) will be determined respectively prior and post to the treatment. TNFa is involved in inflammation and bone loss[14] and RANKL influences pathological processes of multiple bone metabolic diseases[15] .Thus, these two cytokines will be used to reflect the changes of bone metabolism and inflammatory condition of the patient.

\section{Participant timeline $\{13\}$}

Figure 2 and Figure 3 show the study flowchart and the study time points,respectively.

\section{Sample size $\{14\}$}

Patients with AS will be randomized into two groups in a 1:1 ratio. The sample size for each group was calculated according to the following equation for a design with repeated measures [16]

$$
n_{c}=\frac{\left[p_{t}\left(1-p_{t}\right)+p_{c}\left(1-p_{c}\right)\right]\left(\mu_{1-\alpha / 2}+\mu_{1-\beta}\right)^{2}}{\left(p_{t}-p_{c}\right)^{2}}
$$

where $n_{c}$ stands for the number of control group, $p_{t}$ is the clinical effective rate of treatment group,and $p_{c}$ is the clinical effective rate of control group. The values of $a, \mu_{1-\alpha / 2}$, and $\mu_{1-\beta}$ used here are as follows: $a=$ 0.05 (bilateral); $\mu_{1-\alpha / 2}=1.96 ; \mu_{1-\beta}=0.84$. As a result, an estimated sample size of 12 patients per group was obtained, assuming that the effective rate is $97 \%$ in the treatment group and $53 \%$ in the control,based on data from clinical trials reported by the literature[17]. 15 subjects will be needed by each group, considering a possible drop-out rate of about $20 \%$ due to potential eliminations or drop-outs of subjects during the Trial. The Study may differ in manipulation methods and the selection of acupoints, in comparison with those reported in literatures, due to fewer clinical studies reported on thunder-fire 
moxibustion in treating AD. Therefore, the sample size is enlarged to 60 subjects in total with 30 for each group, in order to minimize the error.

\section{Recruitment \{15\}}

Patients are recruited in the TCM clinic and rheumatism immune clinic of Beijig Luhe hospital Affiliated to Capital Medical University.Our annual clinic amount exceeds 130000 people, which provided guarantee for recruit patients.

\section{Assignment of interventions: allocation}

\section{Sequence generation $\{16 a\}$}

Patients will be randomized into 2 parallel groups, with 30 patients in each group.stratified using SPSS 20.0 software (SPSS Inc., Chicago, IL).

\section{Concealment mechanism \{16b\}}

The randomization will be kept in the opaque envelops numbered sequentially from 1 to 60 according the random digits. When a patient is enrolled, the envelop with its number corresponding to the sequence of his/her first visit will be opened, and the group contained by the envelop will represent the treatment that he/she will receive.

\section{Implementation $\{16 c\}$}

The third-party personnel not participating in the Study will be responsible for using SPSS 20.0 software (SPSS Inc., Chicago, IL) to generate a random digit table.

\section{Assignment of interventions: blinding $\{17\}$}

\section{Who will be blinded $\{17 \mathrm{a}\}$}

This study compared the effect of thunder-fire moxibustion therapy with conventional medicine. As it is easy to know whether thunder-fire moxibustion treatment was performed, it is unable to blind the patients and clinical practitioners. However, data collectors and statisticians were blinded in order to eliminate potential bias.

\section{Procedure for unblinding if needed $\{17 b\}$}

The trial design is unable to blind the patients and clinical practitioners, therefore there is no unblinding procedure.

\section{Data collection and management}

Plans for assessment and collection of outcomes $\{18 a\}$ 
Participants will complete standardized surveys prior to the treatment, 2 weeks post to the treatment, and at the end of the treatment. Study staff will complete three standardized, paper-based data collection forms. These forms document the following: TCMSS based on TCM syndrome differentiation in Guiding Principles on Clinical Researches on Novel TCM Drugs in Treating Ankylosing Spondylitis (Draft).BASDAI and BASFI, which will be evaluated according to guideline formulated by International Ankylosing Spondylitis Evaluation Working Group in 2001. Observation will be performed by a fixed researcher during the study.

TNF-a and RANKL will be determined respectively prior and post to the treatment. $5 \mathrm{~mL}$ of peripheral venous blood from elbow will be sampled prior and post to the treatment respectively in the morning from fasted patient, and centrifuged (1800r/min) in ultra-low temperature. The supernatant will be pipetted into an Ependoff tube and kept in a $-80^{\circ} \mathrm{C}$ refrigerator until the unified determination. Enzyme linked immunosorbent assay (ELISA) will be used to determine level of TNF-a and RANKL. Laboratory tests are performed by the clinical laboratory of Beijing Luhe Hospital Affiliated to Capital Medical University .

All researchers, including the acupuncturists, outcome assessors, data collectors, data managers, data entry personnel and statisticians, will undergo training before performing standard procedures and data management. All data will be anonymized, and saved in a encryption study folder.Only the study team has access to this specific study folder.

\section{Plans to promote participant retention and complete follow-up \{18b\}}

The patients will receive initial study information and have at least 2 weeks of reflection at the start of the study.Patients are allowed to stop at any time during the study and are not obliged to give a reason to discontinue.We will distribute a 100 RMB gift to each participant at the completion our study.Throughout the study period, the researchers will keep in close contact with the participants if necessary.If a participant chooses to discontinue the study, the data collected up to the withdrawal date will be anonymized and used.

\section{Data management $\{19\}$}

All data will be managed by epidata(version 3.1 http://www.epidata.dk/ ).After finishing all the surveys ,data will be checked by trained pernonnel,two pople both procrssing data to ensure data accuracy.

\section{Confidentiality $\{27\}$}

All participants will be anonymously enrolled in this study,and a specific alpha-numeric code will be attributed to each subject after enrolment.All paper-based data collection instrumentsand study-related forms will be maintained by storing in locked filing cabinets of study investigators. The electronic data will be saved in a encryption study folder.Only the study team has access to this specific study folder.

Plans for collection, laboratory evaluation, and storage of biological specimens for genetic or molecular analysis in this trial/future use $\{33\}$ 
$5 \mathrm{~mL}$ of peripheral venous blood from elbow will be sampled prior and post to the treatment respectively in the morning from fasted patient, and centrifuged $(1800 \mathrm{r} / \mathrm{min})$ in ultra-low temperature. The supernatant will be pipetted into an Ependoff tube and kept in a $-80^{\circ} \mathrm{C}$ refrigerator until the unified determination.

\section{Statistical methods Statistical methods for primary and secondary outcomes $\{20 \mathrm{a}\}$}

All data will be analyzed using SPSS 20.0 software (SPSS Inc., Chicago, IL). The results of continuous variables will be expressed by mean, standard deviation and $95 \%$ confidence interval; Measurement data will be described with mean \pm standard deviation $(x \pm S D)$, and count data will be expressed with number of cases and percentages. All statistical analyses will use two-tailed tests, and the level of significance will be set at $p<0.05$

Demographic and baseline characteristics of study participants by randomization group will be analyzed by an independent-sample t test or nonparametric test. Data from the primary outcomes (BASDAI score] BASFI score and TCMSS) and secondary outcomes(TNF-aRRANKL) will be presented as continuous variables. In the comparision between the treatment group and control group,the primary outcomes and secondary outcomes will adapt the method of independant -sample test or nonparametric test to analysis the efficacy of thunder-Fire moxibustion in treating AS and its influence on bone metabolism.The relationship between treatment course and therapentic effect in treating AS with thunder-Fire moxibustion will be evaluated by the BASDAI score-BASFI score and TCMSS measured at T1 and T2 in treatment group.They will be analyzed by the Paired-samples' t-test or nonparametric test.Assessment of the presence of TCM syndrome efficacy will be performed with Chi-square test.

\section{Interim analyses $\{21 \mathrm{~b}\}$}

There are no interim analyses planned.

\section{Methods for additional analyses (e.g., subgroup analyses) \{20b\}}

There are no subgroup analyses planned.

\section{Methods in analysis to handle protocol non-adherence and any statistical methods to handle missing data $\{20 \mathrm{c}\}$}

The main comparison and analysis will be performed for outcome indexes based on intention-to-treat set and per-protocol set.

\section{Plans to give access to the full protocol, participant-level data, and statistical code $\{31 \mathrm{c}\}$}

Study-related materials and study-related data would be made available upon request and with permission and approval from the Beijing Luhe Hospital Affiliated to Capital Medical University All requests must be reasonable. 


\section{Oversight and monitoring}

\section{Composition of the coordinating center and trial steering committee $\{5 \mathrm{~d}\}$}

The study will be monitored by an independent internal monitor. The study principal investigator and two co-investigators will be responsible for monitoring and managing data quality, assess completeness and accuracy of data collection,implementation and adherence to the study protocol,and measurement of outcomes.Any decisions needing to be taken regarding the study will be done with the consensus of the entire study team and the Ethics Committee of Beijing Luhe Hospital Affiliated to Capital Medical University will be notified.

\section{Composition of the data monitoring committee, its role, and reporting structure $\{21$ a $\}$}

There are no data monitoring committee in this study.

\section{Adverse event reporting and harms $\{22\}$}

Any adverse events such as fainting, scalded, and local infection during thunder-Fire moxibustion will be recorded and analyzed.

\section{Frequency and plans for auditing trial conduct $\{23\}$}

The Ethics Committee of Beijing Luhe Hospital Affiliated to Capital Medical University is the Trial Steering Committee and will supervise the trial, and the committee will meet once per year.

\section{Plans for communicating important protocol}

\section{amendments to relevant parties (e.g., trial participants, ethical committees) $\{25\}$}

All protocol modifications or amendments will be reported (submitted) to the Ethics Committee of Beijing Luhe Hospital Affiliated to Capital Medical University. Participants will be notified of the amendments or modifications that impact participation, confidentiality, or safety.

\section{Dissemination plans $\{31$ a $\}$}

Results of this research will be disclosed completely in international peer-reviewed journals. Both positive and negative results will be reported.

\section{Discussion}

AS belongs to the domain of arthromyodynia, as considered by classical TCM, and is also called dyphosis or spinal arthralgia. It is stated in Adhering to TCM and Referring to Western Medicine that "all low back pains in human manifest the pain of the spine, which is dominated by the governor meridian... anyone with kidney deficiency must have deficiency in the governor meridian, and this is the main cause of low back pain", indicating the close relationship between the occurrence of low back pain with kidneys 
and the governor meridian. This argument seems consistent with the understanding of AS pathogenesis by many contemporary medical experts[18].It is stated in "On Generative Qi Communicating with Nature of Plain Questions" that dyphosis is generated due to invasion by Cold in the case of failure in opening and closing of Yang Qi. Cold is considered to be the important cause of AS. Kidney stores essence, dominates bone and generates marrow, so the robustness of bone correlates closely to sufficiency of kidney essence. In addition, kidney is congenital origin, containing Nephroyin and Nephroyang of human being, and nourishing/warming human tissues and organs. The governor meridian governs Yang Qi of the whole body and is the sea of all Yang meridians. Moreover, the spine is located at the main road run through by governor meridian and dependent upon the prosperity and nourishment of this meridian. The bone will lose its nourishment in case of Yang deficiency in renal governor, and then the cold evil may take the advantage of weak points and invade into body. Evils both outside and inside the body will joint together, causing failure in transformation of Yang Qi. The prevalence of cold evil goes deep into bones and joints and stays at the spine, manifesting stiffness and pain at the lumbar spine, so that this disease develops. In summary, the main pathogenesis of this disease is renal deficiency with governor meridian cold.

Thunder-fire moxibustion, developed on the basis of ancient thunder-fire miraculous needle, comprises multiple drugs in specific ratios including Artemisia argyi, Frankincense, Myrrh, Aquilaria, Ramulus Cinnamomi, notopterygium Incisum, etc[19].Its thermal power is strong with powerful thermal radiation while burning, capable of forming a large infrared web with a stronger penetration than that of ordinary moxibustion[20]. Medicine molecules inside the moxa stick adhere to the skin and form a region with high concentration of drugs around the lesion, and then penetrate into deep tissues through thermal effect. Thunder-fire moxibustion targeting at the spinal segment of the governor meridian may improve local circulation of Qi and blood, eliminate cold and stop pain, warm meridians and facilitate Yang, and disperse the humidity and dredge meridians, which is compatible with the main pathogenesis of kidney deficiency with governor meridian cold, thereby exerting fully the advantages of thunder-fire moxibustion including powerful heat, strong penetration and good analgesic effect, and achieving the aim of direct arrival at the disease with accelerated onset of action.

From the point of view of modern medicine, the typical pathological change of AS includes inflammatory bone destruction and osteogenesis[21]. An infiltration with a great deal of inflammatory cells exists in bones, joints and synovial tissues in AS patients, and cytokines excreted by these cells play an important role in the chronic inflammatory process and during bone destruction and osteogenesis in AS patients[22]. As shown by researches, the proinflammatory cytokine TNFa is involved in inflammation and bone loss[14], and a great importance attached to earlier effective anti-inflammatory treatment may relieve inflammatory bone destruction in AS patients, and reduce the incidence of hyperosteogeny and arthrodesis[23]. Zeyun Yu et al[24] used conventional drugs for treating rheumatoid arthritis in combination with moxibustion, and the results showed that moxibustion could reduce the content of IL$1 \beta a n d$ TNF- $\alpha$ in serum of the patients, thereby alleviating their synovial symptoms. Similar conclusions were also obtained by the research by Luo Ji et al[25]. RANKL not only regulates biological process of bone metabolism, but also influences pathological processes of multiple bone metabolic diseases, 
especially the formation, survival and activation of osteoclasts[15]. It has been proven by results from animal experiments by Yang Chen[26] that moxibustion can lower the expression of rankl mRNA and increase the expression of opg mRNA, suggesting a recovery of balance between osteoblasts and osteoclasts, based on which moxibustion is considered capable of alleviating cartilage degradation and bone destruction in rabbit RA model possibly through modifying OPG/RANKL signaling pathway. In summary, moxibustion may ameliorate systemic inflammation and bone metabolism disorder, and its mechanism may be related to the capability of moxibustion in regulating content of some systemic inflammatory factors and cytokines involved in bone metabolism. Further studies are justified to explore whether the goal to ameliorate abnormal bone metabolism in AS patients can be achieved by thunder-fire moxibustion through the same route.

The Study aims primarily to explore the efficacy of thunder-fire moxibustion on patients with AS of kidney deficiency and governor meridian cold and its influence on bone metabolism, enrolling AS patients as the subjects randomized into the treatment group and the control treated respectively with drugs plus thunder-fire moxibustion and with drugs alone. The clinical efficacy of thunder-fire moxibustion in AS patients with kidney deficiency with governor meridian cold will be verified based on the evaluation of indexes of clinical efficacy and related cytokines prior and post to thunder-fire moxibustion, and the mechanism will be discussed preliminarily for this indication of thunder-fire moxibustion. The design is rigorous and capable of providing more evidences for thunder-fire moxibustion in treating AS.

\section{Abbreviations}

AS:ankylosing spondylitis;TCM:Traditional Chinese Medicine;BASDAl:Bath Ankylosing Spondylitis Disease Activity Index;BASFI:Bath Ankylosing Spondylitis Functional Index;TCMSS:Traditional Chinese Medicine syndrome score;TNF-a:tumor necrosis factor-a;RANKL: receptor activator of nuclear factor-K $B$ ligand

\section{Declarations}

\section{Trial status}

The current protocol is version 2 of 21-1-2021.Participant recruitment began on March 20, 2020, and will end on approximately April 30,2022.

Additional files

Additional file 1:TCM symptom score scale. (DOCX $14 \mathrm{~kb}$ )

Additional file 2: BASDAI score scale . (DOCX $13 \mathrm{~kb}$ )

Additional file 3: BASFI score scale . (DOCX 14 kb) 
Acknowledgements

We would like to acknowledge all patients and doctors who will participate in this trial for their cooperation and the funding support agencies for agreeing to sponsor the study.

\section{Authors' contributions $\{31 \mathrm{~b}\}$}

ML and YL conceived and designed the study protocol and approved the final version; . YL wrote the manuscript. PW participated in the design of this protocol. YYS, JQ collected the data and coordinated the recruitment and treatment of patients. All authors read and approved the final manuscript.

\section{Competing interests $\{28\}$}

The authors declare that they have no competing interests.

\section{Funding $\{4\}$}

The trial is funded by the foundation for TCM development of science and technology of Beijing,Beijing Traditional Chinese Medicine Administration Bureau,China(JJ-2020-56). The funder provided research funding but had no role in the design or execution of the trial.

\section{Availability of data and materials $\{29\}$}

The dataset will be available upon reasonable request once the results have been published.

\section{Ethics approval and consent to participate $\{24\}$}

The study will be carried out in accordance with the current version of the Declaration of Helsinki and approved by the Ethics Committee of Beijing Luhe Hospital Affiliated to Capital Medical University (2020LHKY-067-02). Written, informed consent to participate will be obtained from all participants. If the participant is unable toprovide consent, their family members, an authorized representative, or a neutral physician may provide consent until the participant is able to do so.

\section{Ethics approval and consent to participate $\{24\}$}

Informed consent will be obtained from all the study participants before they are enrolled in the study. The participants will be allowed to withdraw from the study at any time during the study period without reason. This research adhered to the principles of the Declaration of Helsinki, was approved by the Ethics Committee of Beijing Luhe Hospital Affiliated to Capital Medical University (2020-LHKY-067-02) on February 05, 2021, and was registered with the China Clinical Trials Registry (ChiCTR2100044227) on March 12, 2021 (http://www.chictr.org.cn)

\section{Consent for publication $\{32\}$}

Not applicable. 


\section{Author details}

${ }^{1}$ Beijing Luhe Hospital Affiliated to Capital Medical University, Beijing 101149, China.

\section{References}

1. Rheumatology branch of Chinese. Medical Association.Guidelines for the diagnosis and management of Ankylosing Spondylitis. Chinese Journal of. Rheumatology.2010;14(08):557-559. doi:CNKI:SUN:FSBZ.0.2010-08-021.

2. Zhu W, He XX, Cheng KY, Zhang LJ, Chen D,Wang X, Qiu GX,et al. Ankylosing spondylitis: Etiology, pathogenesis, and treatments.Bone Research. 2019;7:22. doi: 10.1038/s41413-019-0057-8.

3. Wang Runsheng,Ward Michael M.Epidemiology of axial spondyloarthritis:an update.Curr Opin Rheumatol. 2018; 30(2): 137-143.

4. Yu YY,Mao JC.Progress of the Treatment of Ankylosing Apondylitis.Jilin. Journal of Traditional Chinese Medicine.2019;39(01):136-139.doi:

10.13463/j cnki.jlzyy.2019.01.038.

5. Chang H, Liang Jk, Li H, Liu Jt Yu, My. Zhao Bx. Moxibustion in Early Chinese Medicine and Its Relation to the Origin of Meridians: A Study on theUnearthed. LiteraturesEvid Based Complement Alternat Med. 2017. https://doi.org/10.1155/2017/8242136.

6. Zhong YM, Cheng B, Zhang LL, Lu WT, Shang YN,Zhou HY.Effect of Moxibustion on Inflammatory Cytokines in Animals with Rheumatoid Arthritis: A Systematic Review and Meta-Analysis.Evid Based Complement Alternat Med. 2020.;2020:6108619.doi:10.1155/2020/6108619.

7. Qi Q, Liu YN, Jin XM, Zhang LS, Wang C, Bao CH, Liu HR, Wu HG. Wang XM.Moxibustion treatment modulates the gut microbiota and immune function in a dextran sulphate sodium-induced colitis rat model. World J Gastroenterol. 2018;24(28):3130-44.

8. Gong YY, Yu ZY, Wang YN, Xiong Y, Zhou YM, Liao CX, et al. Effect of Moxibustion on HIF-1a and VEGF Levels in Patients with Rheumatoid Arthritis. Pain Res Manag. 2019. doi:10.1155/2019/4705247.

9. Hui X, Wang H, Yao Q, Zhao BX, Ha L.Moxibustion therapy for treating patients with primary osteoporosis A systematic review and meta-analysis protocol.Medicine (Baltimore). 2019; doi: 10.1097/MD.0000000000018226.

10. Hu JJ, Mao YY, Zhang Y, Ye D, Wen CP, Xie ZJ. Moxibustion for the treatment of ankylosing spondylitis: a systematic review and meta-analysis. Ann Palliat Med. 2020;9(3):709-20.

11. Zhu D. Gao ML,Yao JS.Clinical research progresses of moxibustion in the treatment of ankylosing spondylitis. Rheumatism Arthritis. 2018;7(02):77-80. doi:CNKI:SUN:FSBG.0.2018-02-025.

12. Huang RN, Huang YX, Huang RJ, Huang SF, Wang XJ, Yu XJ,et al. Thunder-Fire Moxibustion for Cervical Spondylosis: A Systematic Review and Meta-Analysis. Evid Based Complement Alternat Med. 2020. doi:10.1155/2020/5816717. 
13. Xu DM, Xu HN, Liu J, Wang T, Wu WZ, Liu LY, et al. Effect of Thunder-Fire Moxibustion on Pain, Quality of Life, and Tension of Multifidus in Patients with Primary Osteoporosis: A Randomized Controlled. Med Sci Monit. 2018. doi:10.12659/MSM.909725.

14. Kimberley Johanna Beek,Tamara Rusman,Maria Alida Cornelia van der Weijden,Willem Frederik Lems,Johannes Christiaan van Denderen,Maria Konsta,et al.Long-Term Treatment With TNF-Alpha Inhibitors Improves Bone Mineral Density But Not Vertebral Fracture Progression in Ankylosing Spondylitis.Journal of Bone and Mineral Research,2019;doi: 10.1002/jbmr.3684.

15. Takehito O,Mikihito Hayashi,Fumiyuki Sasaki,Tomoki Nakashima.RANKL biology: bone metabolism, the immune system, and beyond.Inflamm Regen.2020; doi: 10.1186/s41232-019-0111-3.

16. Lv YQ. Feng GS.Common Method on Sample Size Calculation in Medical Research. Chronic Pathematology J. 2016;17(04):359-61. doi:10.16440/j.cnki.1674-8166.2016.04.001.

17. Du TT. Efficacy of Thunder-Fire Moxibustion on stimulating Huatu Jiaji acupoint in Treating Ankylosing Spondylitis of Kidney Deficiency and Governor Meridian Cold.Nanjing Medical University, 2017. https://kns.cnki.net/KCMS/detail/detail.aspx?

dbname=CMFD201801\&filename $=1018042556$.nh.

18. Ding FR, Jiang P, Liu W. The Progress of Diagnosis and Treatment of Ankylosing Spondylitis in TCM.Shandong. J Tradit Chin Med. 2019;38(08):805-8. doi:10.16295/j.cnki.0257-358x.2019.08.023.

19. Zhao SZ.Thunder-Fire Moxibustion therapy in China.In:Chen XH,Chen QX,editors.Shanghai Far East Publishers.Shanghai:TCM Clinical;2008. p. 46.

20. Zheng XH. He TT,Ma SG.30 cases of kidney deficiency-cold syndrome enuresis children by thunderfire moxibustion. Guangzhou Medical Journal. 2020;51(06):75-8. ..doi:CNKI:SUN:GZYY.0.2020-06016.

21. Li LY, Chen B, Zhao. HY,Wang GS.Bone changes and curative effect of infliximab in patients with ankylosing spondylitis. Journal of Musculoskelet Neuronal Interactions. 2020;20(3):437-443.

22. Sungsin Jo,Sung Eun Wang, Young Lim Lee, Suman Kang, Bitnara Lee,Jinil Han, et al.IL-17A induces osteoblast differentiation by activating JAK2/STAT3 in ankylosing spondylitis. Arthritis Res Ther. 2018; doi: 10.1186/s13075-018-1582-3.

23. Hsu-Wen Tseng ME, Pitt, Tibor T, Glant, Allan F, McRae TJ, Kenna MA, Brown, et al. Inflammationdriven bone formation in a mouse model of ankylosing spondylitis: sequential not parallel processes. Arthritis Res Ther. 2016;18:35.doi. 10.1186/s13075-015-0805-0.

24. Yu ZY, Wang YN, Li Y, Liao CX,Dai JY, Luo Y, et al. Effect of Moxibustion on the Serum Levels of MMP-1, MMP-3, and VEGF in Patients with Rheumatoid Arthritis. Evid Based Complement Alternat Med. 2020. doi:10.1155/2020/7150605.

25. Luo J, Liu S. Effect of Heat-Sensitive Moxibustion on Pain and Expressions of TNF-a and IL-1 in Patients with KOA. Journal of Clinical Acupuncture Moxibustion. 2020;36(06):55-8.

26. Chen Y, Li HJ, Luo XC, Liu HH, Zhong YM, Wu X,et al. Moxibustion of Zusanli (ST36) and Shenshu (BL23) Alleviates Cartilage Degradation through RANKL/OPG Signaling in a Rabbit Model of Rheumatoid Arthritis. Evid Based Complement Alternat Med. 2019. doi:10.1155/2019/6436420. 
Figures

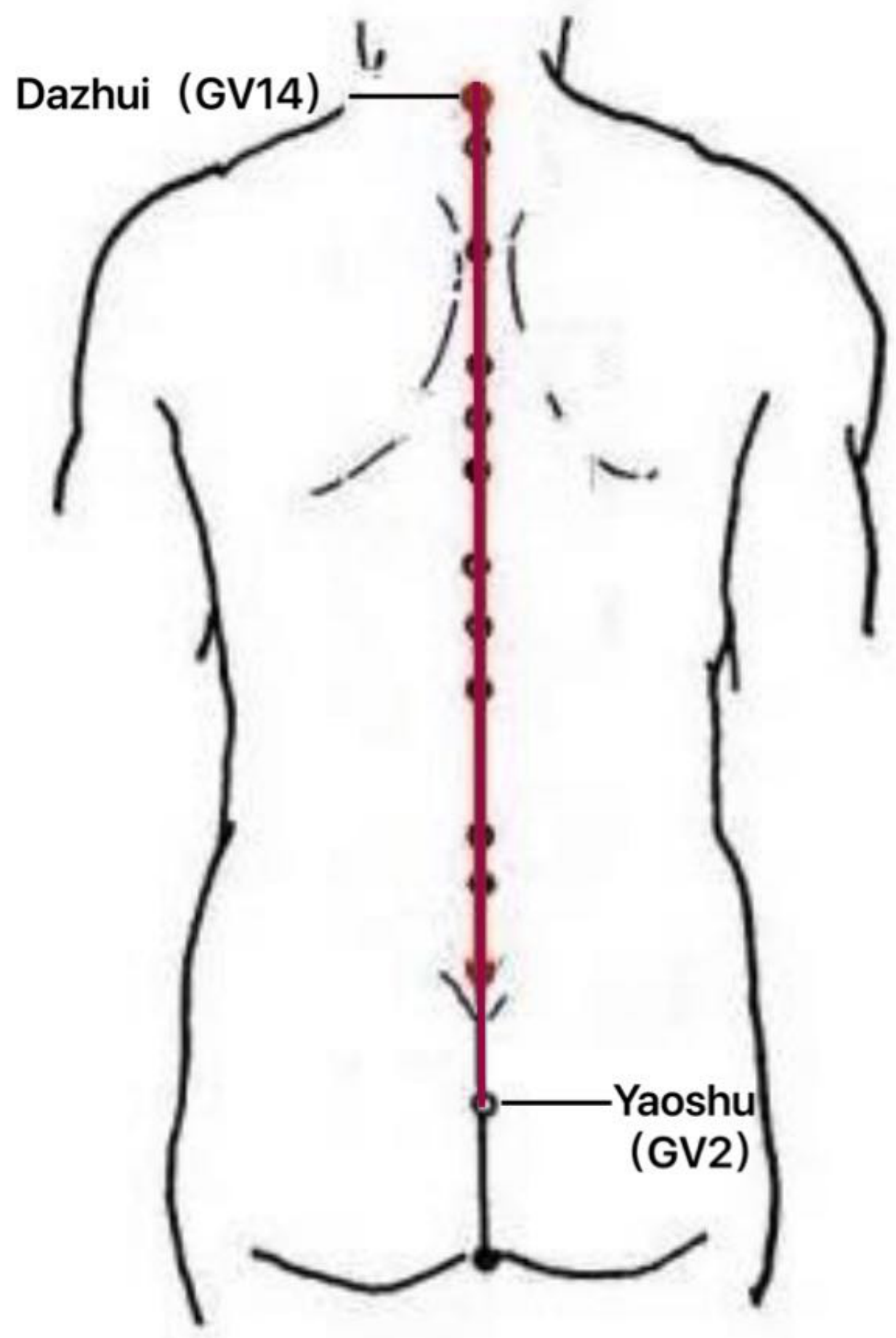

Figure 1

The sites for thunder-fire moxibustion. The sites will be selected along the governor meridian in its spinal segment, with the acupoints Dazhui and Yaoshu as the border. 


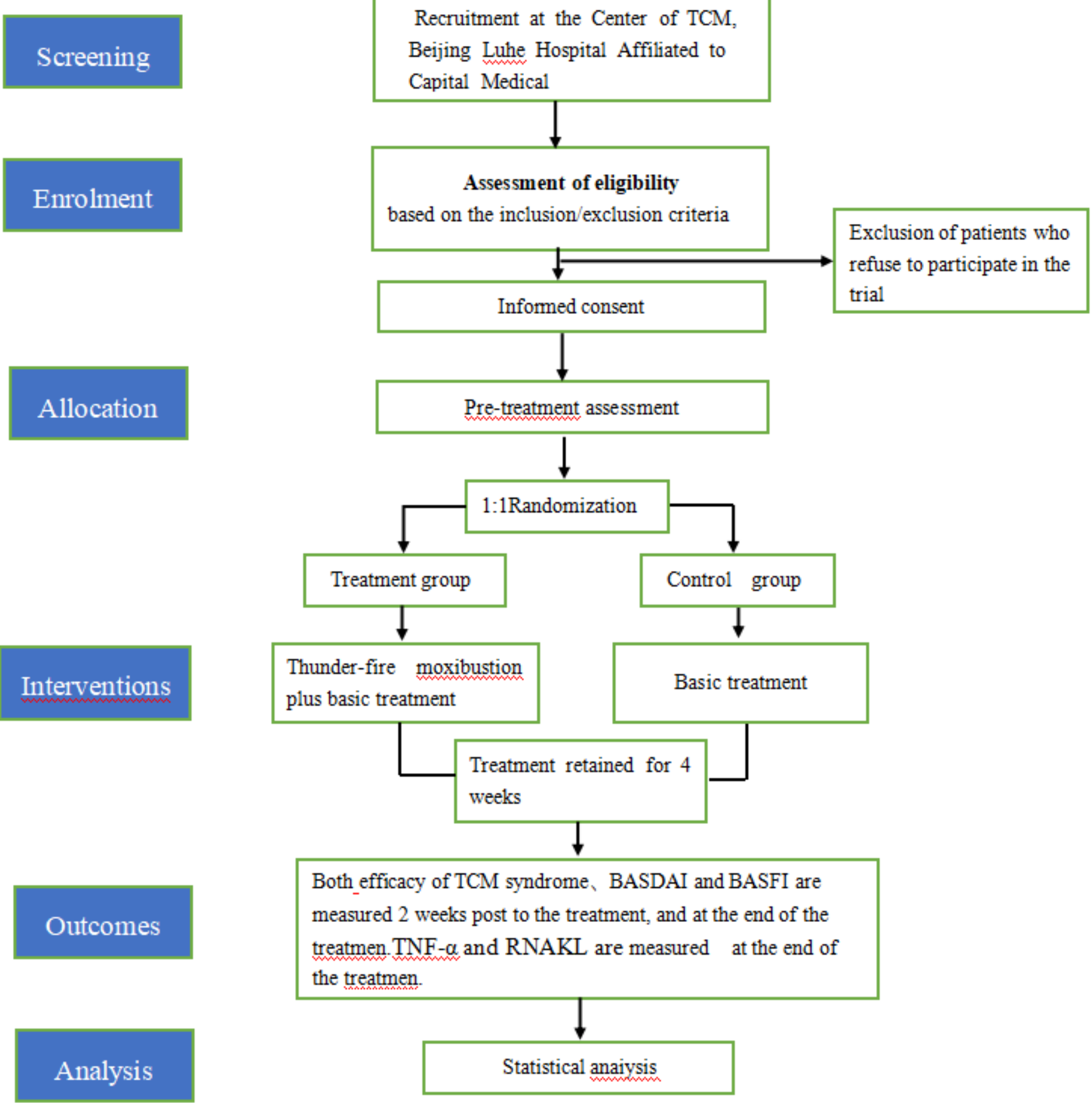

\section{Figure 2}

Flow chart of the trail, TCM traditional Chinese medicine,BASDAl Bath Ankylosing Spondylitis Disease Activity Index,BASFI Bath Ankylosing Spondylitis Functional Index,TNF-a tumor necrosis factor-a, RNAKL receptor activator of nuclear factor-kB ligand 


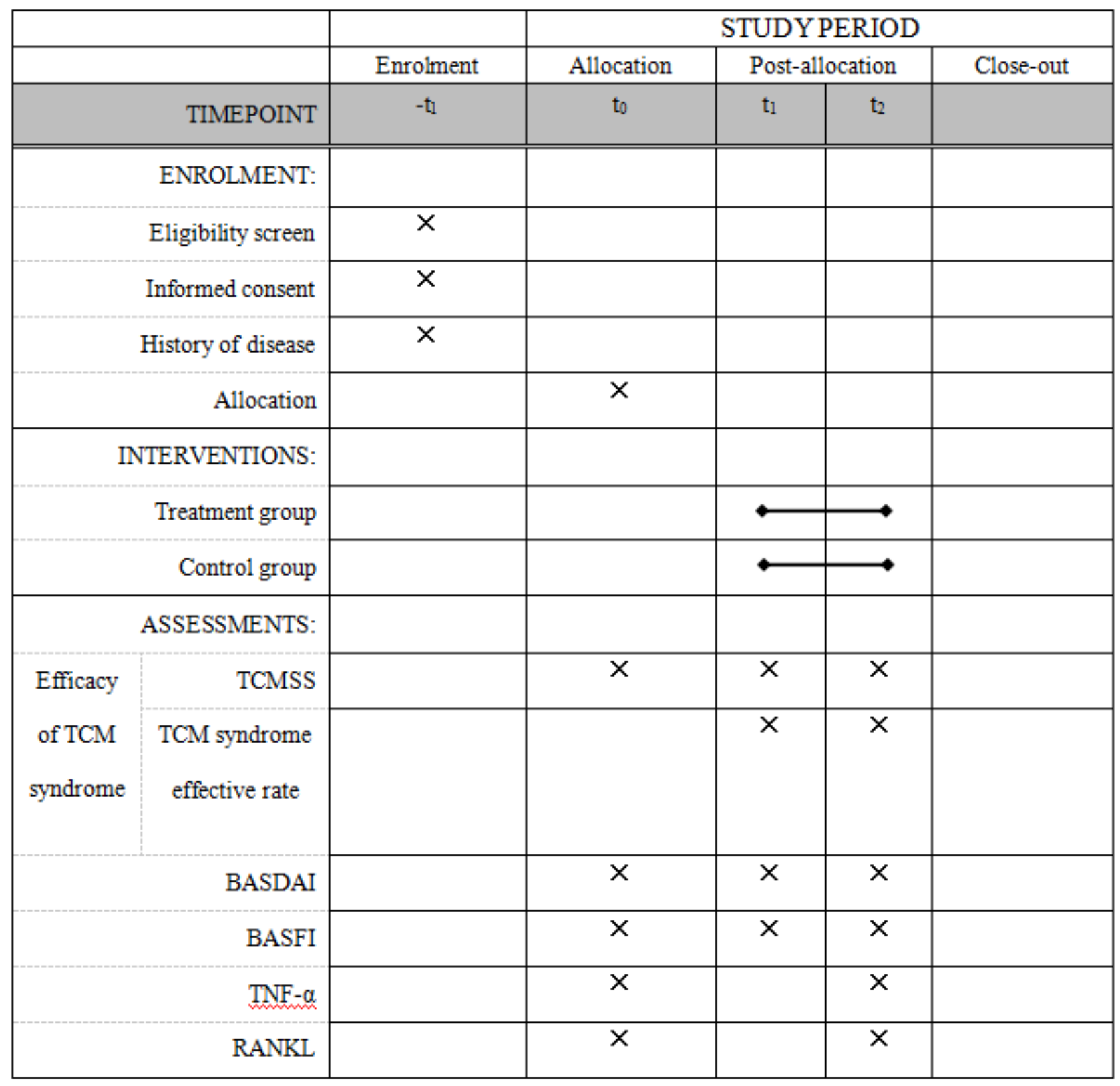

Figure 3

SPIRIT figure

\section{Supplementary Files}

This is a list of supplementary files associated with this preprint. Click to download.

- TCMsymptomscorescale.docx

- BASDAlscorescale.docx

- BASFIscorescale.docx 